\title{
Study Protocol for the Effects of Artificial Intelligence (AI)-Supported Automated Nutritional Intervention on Glycemic Control in Patients with Type 2 Diabetes Mellitus
}

\author{
Rie Oka (1) - Akihiro Nomura · Ayaka Yasugi • Mitsuhiro Kometani · \\ Yuko Gondoh · Kenichi Yoshimura · Takashi Yoneda
}

Received: February 4, 2019 / Published online: March 15, 2019

(C) The Author(s) 2019

\begin{abstract}
Introduction: Nutritional intervention is effective in improving glycemic control in patients with type 2 diabetes but requires large inputs of manpower. Recent improvements in photo analysis technology facilitated by artificial intelligence (AI) and remote communication technologies have enabled automated evaluations of nutrient intakes. AI- and mobile-
\end{abstract}

Enhanced Digital Features To view enhanced digital features for this article go to https://doi.org/10.6084/ m9.figshare.7798436

R. Oka ( $($ ) · A. Nomura · M. Kometani · Y. Gondoh . T. Yoneda

Department of Cardiovascular and Internal

Medicine, Kanazawa University Graduate School of

Medical Science, Kanazawa, Japan

e-mail: ririoka@goo.jp

R. Oka

Department of Internal Medicine, Hokuriku Central Hospital, Toyama, Japan

A. Nomura $\cdot$ K. Yoshimura

Innovative Clinical Research Center (iCREK),

Kanazawa University Hospital, Kanazawa, Japan

A. Nomura

CureApp Institute, Karuizawa, Japan

A. Yasugi

Asken Medical Division, WIT Co., Ltd., Tokyo, Japan

T. Yoneda

Institutes of Liberal Arts and Science, Kanazawa

University, Kanazawa, Japan supported nutritional intervention is expected to be an alternative approach to conventional in-person nutritional intervention, but with less human resources, although supporting evidence is not yet complete. The aim of this study is to test the hypothesis that AI-supported nutritional intervention is as efficacious as the in-person, face-to-face method in terms of improving glycemic control in patients with type 2 diabetes.

Methods: This is a multicenter, unblinded, parallel, randomized controlled study comparing the efficacy of AI-supported automated nutrition therapy with that of conventional human nutrition therapy in patients with type 2 diabetes. Patients with type 2 diabetes mainly controlled with diet are to be recruited and randomly assigned to AI-supported nutrition therapy $(n=50)$ and to human nutrition therapy $(n=50)$. Asken, a mobile application whose nutritional evaluation has been already validated to that by the classical method of weighted dietary records, has been specially modified for this study so that it follows the recommendations of Japan Diabetes Society (total energy restriction with proportion of carbohydrates to fat to protein of 50-60, 20, and 20-30\%, respectively).

Planned Outcomes: The primary outcome is the change in glycated hemoglobin levels from baseline to 12 months, and this outcome is to be compared between the two groups. The secondary outcomes are changes in fasting plasma 
glucose, plasma lipid profile, body weight, body mass index, waist circumference, blood pressures, and urinary albumin excretion. The results of this randomized controlled trial will fill the gap between the demand for support of $\mathrm{AI}$ in nutritional interventions and the scientific evidence on its efficacy.

Trial Registration: UMIN000032231.

Keywords: Artificial intelligence (AI); Behavior change; Nutritional therapy; Remote healthcare

\section{INTRODUCTION}

Successful diabetes care requires not only the proper medications but also lifestyle modifications in terms of diets and activity [1,2]. Among the variety of weight loss methods and multifaceted interventions on lifestyle, nutritional intervention has the largest statistically significant effect on glycemic control in patients with type 2 diabetes [3]. Due to the progressive nature of type 2 diabetes [4], early nutritional interventions, such as before insulin injections are needed, may be very effective [5]. Prior studies demonstrating significant decreases in glycosylated hemoglobin (HbA1c) during a 1-year nutritional intervention involved frequent contact between patients and registered dietitians, with, for example, patients seen individually on a weekly basis in the Look AHEAD trial [6] and on a once-monthly basis in the MÈDITA Randomized Trial [7]. Such intense interventions are difficult to maintain in clinical settings because both patients and providers report lack of time [8] and healthcare resources are limited [9].

Recent technologies for remote healthcare has a potential to reduce the burden of both patients and healthcare providers regarding dietary management $[10,11]$. Patients can make daily dietary records using mobile devices and communicate online with dieticians without the need for hospital visits. Dietary self-monitoring in itself is considered to be an effective strategy for effecting behavior change [12], and adherence to dietary self-monitoring is higher when an electronic diary is used than when paper records are kept with or without feedbacks [13]. Moreover, improvements in photo analysis technology that often includes artificial intelligence AI) has enabled the automatic identification of food items and evaluation of their nutritional values, by which realtime dietary feedbacks are provided [14]. AIand mobile-supported nutritional intervention is expected to overcome time and location barriers in human interventions and boost the motivation of patients with type 2 diabetes to adhere to their diet.

With this background, our aim is to demonstrate the efficacy of automated nutritional intervention by use of a mobile phone and AI on reducing glycemia in patients with type 2 diabetes. In the study described here, we adopt a mobile application whose nutritional evaluation has been already validated to that by the classical method of weighted dietary records [15]. The hypothesis of this study is that AIsupported nutritional intervention is as efficacious as in-person, face-to-face method in improving glycemic control in patients with type 2 diabetes.

\section{METHODS}

\section{Study Design}

This is a multicenter, unblinded, parallel, randomized controlled study aimed at comparing the efficacy of AI-supported automated nutrition therapy with that of conventional human nutrition therapy in patients with type 2 diabetes. The study protocol was approved by the Institutional Review Board of Kanazawa University on April 13, 2018 (IRB no. 2623-3); all recruited patients are required to provide written informed consent. This trial is registered in the University Hospital Medical Information Network (UMIN-CTR; https://www.umin.ac.jp/ ctr; UMIN ID: UMIN000032231), and is being conducted in accordance with the Declaration of Helsinki of 1964, as revised in 2013. Patient recruitment commenced on April 13, 2018 and is scheduled to continue through to April 13, 2020 . 


\section{Study Setting and Participants}

Participants are recruited from patients attending the outpatient clinics of the Department of Diabetes and Endocrinology at Kanazawa University Hospital, Hokuriku Central Hospital, and Houju Memorial Hospital. Those patients who are obese or overweight and have type 2 diabetes mainly controlled with diet are eligible.

Inclusion criteria are (1) age $\geq 20$ years; (2) body mass index (BMI) cutoff for Asians of $>23 \mathrm{~kg} / \mathrm{m}^{2}$, which is the World Health Organization cutoff for Asians requiring public health action [16]; (3) physician diagnosis of type 2 diabetes confirmed by clinical data (e.g., documentation of fasting plasma glucose [FPG] of $\geq 126 \mathrm{mg} / \mathrm{dL}$, a positive oral glucose tolerance test of $>200 \mathrm{mg} / \mathrm{dL}$, or $\mathrm{HbA} 1 \mathrm{c} \geq 6.5 \%$ ); (4) owning and using a mobile phone in their daily lives; and (5) able to understand study procedures and provide written informed consent.

Exclusion criteria include (1) use of insulin and/or glucagon-like peptide-1 within the past 6 months; (2) use of agents causing weight loss; (3) HbA1c $\geq 7.5 \%$; (4) renal dysfunction (e.g., urinary protein $>1 \mathrm{~g} / \mathrm{g}$ creatinine and/or an estimated glomerular filtration rate of $<45 \mathrm{~mL}$ / $\mathrm{min} / 1.73 \mathrm{~m}^{2}$ ); (5) uncontrolled thyroid disease; (6) uncontrolled hypertension (diastolic blood pressure $>100 \mathrm{~mm} \mathrm{Hg}$ and/or systolic blood pressure $>160 \mathrm{~mm} \mathrm{Hg}$ ) and/or secondary hypertension; (7) oral or injected steroid use within the past 3 months; (8) pregnancy or planned pregnancy during the study period; (9) history of myocardial infarction, severe uncontrolled heart failure, unstable angina, transient ischemic attack, or stroke in the past 6 months; (10) receiving treatments for malignancies; (11) diagnosed eating disorder; and (12) medical or psychological condition(s) which would compromise the ability to meet protocol requirements in the opinion of the investigator.

\section{Interventions}

Patients are allocated randomly to either AIand mobile-supported nutrition therapy or inperson nutrition therapy by human dieticians for 1 year. The principle of nutrition therapy in both interventions is total energy restriction, based on the recommendations of Japan Diabetes Society (JDS), Japan Atherosclerosis Society (JAS), and Japan Society for the Study of Obesity (JASSO); the ideal body weight (IBW) is calculated as IBW $=$ height $(\mathrm{m})^{2} \times 22\left(\mathrm{~kg} / \mathrm{m}^{2}\right)$ $[17,18]$, and the total dietary energy (kcal/day) is estimated as $25-35 \mathrm{kcal} / \mathrm{kg}$ IBW/day. Physicians determine the total dietary caloric intake according to the daily activity of each patient $[17,18]: 25-30 \mathrm{kcal} / \mathrm{kg}$ IBW for subjects with low-level activity, $30-35 \mathrm{kcal} / \mathrm{kg}$ IBW for subjects with usual-level activity, and $\geq 35 \mathrm{kcal} / \mathrm{kg}$ IBW for subjects with heavy-level activity. The recommended macronutrient distribution is set to be $50-60 \%$ from carbohydrate sources, $\leq 20 \%$ from protein sources, and $20-30 \%$ from fat sources [17].

\section{Experimental Group: AI-Supported Nutrition Therapy}

The mobile phone app used for this study is called "Asken" and is one of the most popular Japanese apps for behavior change among individuals aspiring to lose weight. The criteria of its nutritional feedback system has been specially modified for this study so that it follows the recommendations set by the JDS. One of the main features of this app is the availability of various food-logging interfaces, most notably an AI-powered photo analysis system linked with its extensive database of over 100,000 menus both on the market and homemade. Once a photo of a hamburger combo is taken, for example, the system instantly identifies the frame of each item as well as its menu and serving amount (Fig. 1). After confirmation by the patient, during which process the patient can re-select the correct type or brand of hamburger from 82 types/brands in the database, the app calculates the energy and nutrient intakes based on the Standard Tables of Food Composition in Japan 2015, seventh revised edition [19]. The validity and accuracy of Asken's estimated total energy intake and macronutrients, such as carbohydrates, fat, and protein, have been demonstrated in an earlier 


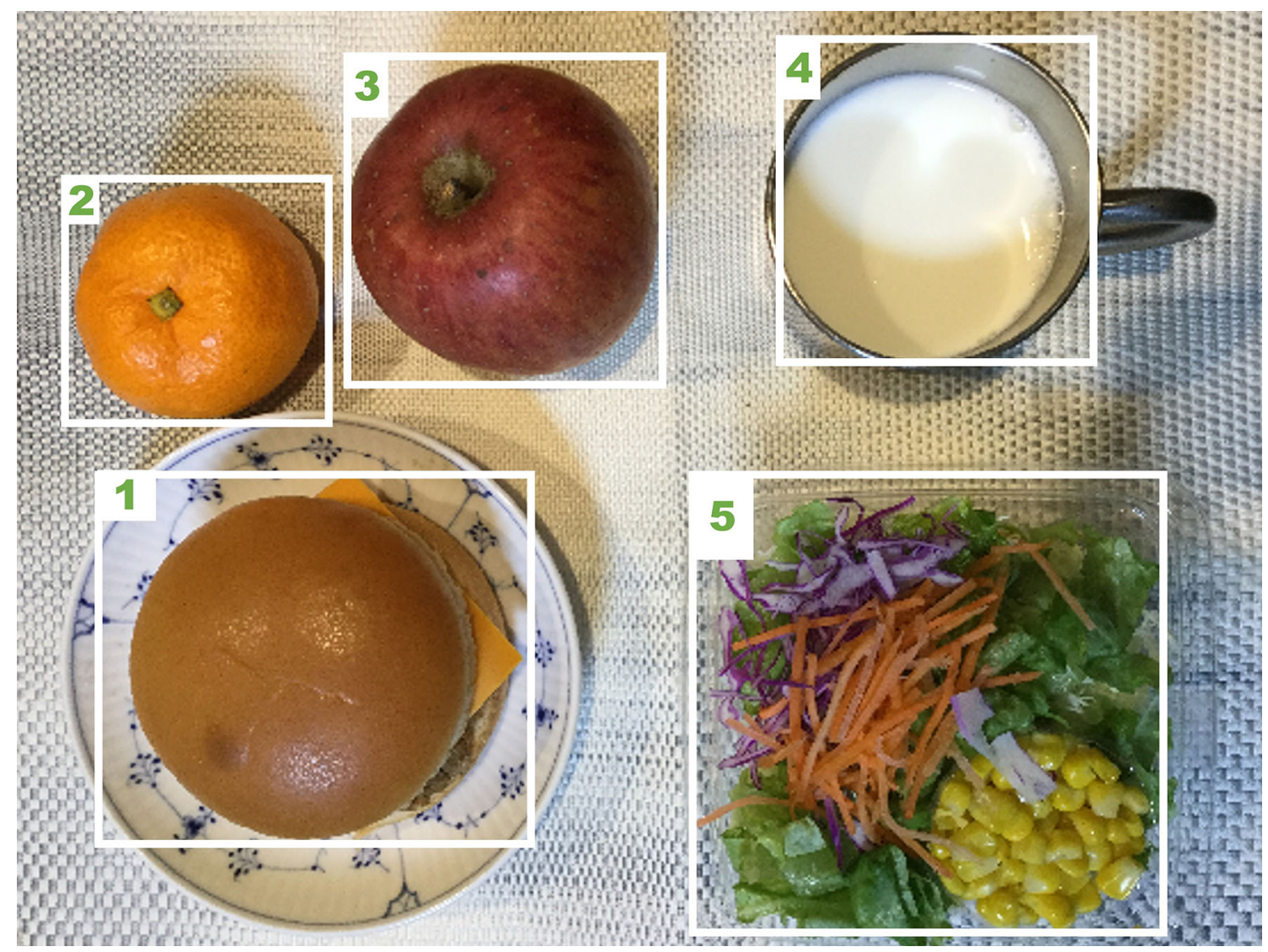

Fig. 1 Artificial intelligence (AI)-powered photo analysis of a meal. Deep learning AI analyzes the photo of the entire meal and identifies the frame of each item as well as its menu and serving amount

study [15]. Following each food logging, Asken eventually shows how the user has satisfied his/ her recommended range of daily intake of each nutrient with a graph (Fig. 2) and delivers an individualized message by AI based on the Dietary Reference Intakes for Japanese 2015 [20]. Because this whole process from menu identification with photo analysis to generation of dietary feedbacks is fully unmanned and automated, the patients can learn their detailed nutritional conditions anywhere and anytime. These dietary messages and feedbacks are also delivered by a female Japanese character "Miki"; there are more than 200,000 different patterns for her wording and facial expressions. "Miki" always remains compassionate and empathetic to the patients even when they eat a lot or skip a recording of food intake (Fig. 3).

The patients also have access to peer support from other Asken users through the in-app diary function. Weights and steps can be automatically recorded in the app by its data-sharing function with other apps, such as GoogleFit and Apple Healthcare. Patients are instructed to log onto the Asken app and input meals and snacks at least once a week.

\section{Comparison Group: Human Nutrition Therapy}

In Japan, nutrition therapy managed by human dietitians is covered by health insurance programs for patients with diabetes. The principle of nutrition therapy of a comparison group is the same as that of the experimental group, namely, total caloric restriction with macronutrient distribution as recommended by the JDS [17]. At baseline, dietitians estimate the current energy and nutrient intakes on a face-to-face 


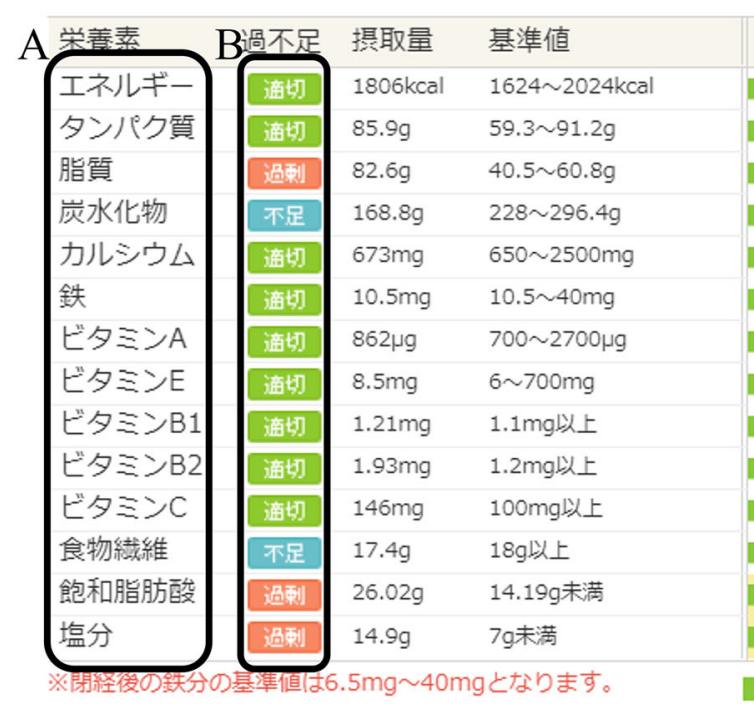

Fig. 2 Evaluation of energy and nutrient intakes. Asken shows how the patient has satisfied his/her recommended range of daily intake of each nutrient with a graph on the basis of data input by the patient. Translation of text in area A (from top to bottom): energy, protein, lipids,

interview. Participants and dietitians work together to design feasible strategies to improve dietary habits. Patients are provided with printed materials for describing their plans and recording their meals. After the first interview at baseline, human dietitians continue to evaluate the achievement status of the initial plans based on the paper records and to provide individual feedbacks once or more times within 3 months. Patients have a one-to-one meeting for $\geq 20 \mathrm{mi}$ with a human dietitian during each visit.

\section{Outcomes}

The primary outcome of this study is the change in HbA1c levels during 1 year of intervention, with the outcomes to be compared between the two groups. HbA1c is an accepted diagnostic parameter by which to monitor long-term glycemic control [21]. Secondary outcomes are absolute changes in (1) FPG; (2) plasma lipid profile, total cholesterol, triglycerides, highdensity lipoproteins, and low-density lipoproteins; (3) body weight, BMI, and waist circumference; (4) systolic and diastolic blood pressure; and (5) urinary albumin excretion. (6) Recorded

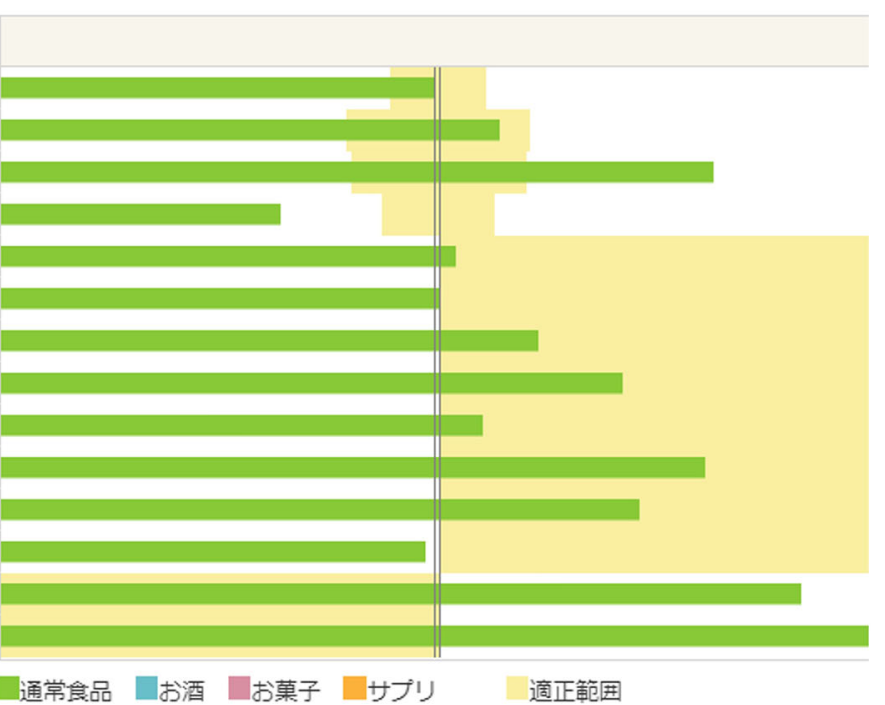

carbohydrates, calcium, iron, vitamin $\mathrm{A}$, vitamin $\mathrm{E}$, vitamin B1, B2, vitamin C, dietary fiber, saturated fatty acids, and salt. In area $\mathrm{B}$, red boxes indicate excessive intake, green boxes indicate appropriate intake, and blue boxes indicate deficiencies

data on the Asken app (frequencies of log-ons, weight, daily intakes of total energy and nutrients) and (7) costs of the interventions in economic terms are also evaluated.

\section{Blood Sampling and Anthropometric Measurements}

Venous blood samples are drawn from the antecubital vein after an overnight fast by a trained nurse and transported to the central laboratory of each hospital for analysis. HbA1c is measured using a high-performance liquid chromatography method. Lipids are measured using enzymatic analytical chemistry methods, and plasma glucose is assessed using the glucose oxidase method. Resting blood pressure is measured in the sitting position with an automatic device after $\geq 5$ min of rest. Measurements of BMI and waist circumference are conducted according to published methods [22]. 


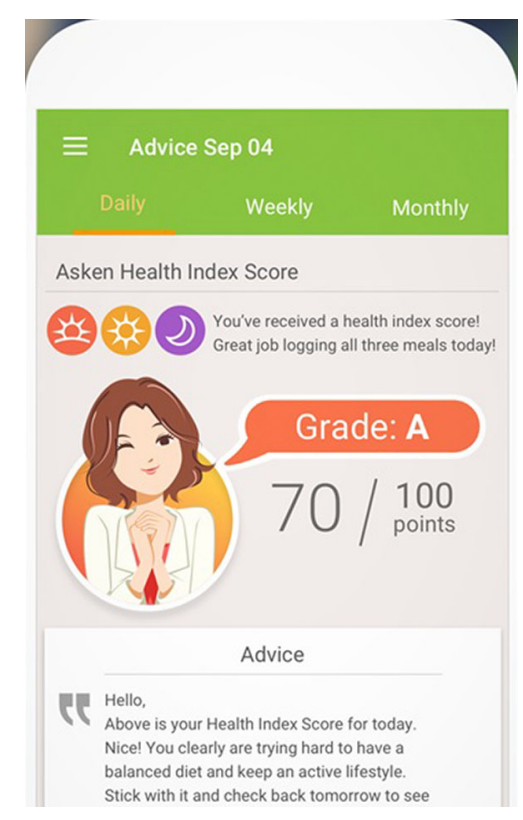

Fig. 3 Dietary messages and feedbacks which appear on Asken. Feedbacks are delivered by a female Japanese character "Miki," who is always compassionate and empathetic with patients. This figure is taken from the US version of Asken app only for reference; the study is conducted with the Japanese version

\section{Concomitant Medication}

In principle, treatments for diabetes, hypertension, and dyslipidemia are not changed during the study period in order to avoid effects on the efficacy assessments of this study. If it is inevitable to add a new antidiabetic drug to the treatment regimen, a full description of the change and the reason and timing are described in the case report form (CRF). If insulin therapy is started, the trial will be discontinued at that time.

\section{User Privacy}

User privacy is preserved from two directions, the app and humans. First, the app is equipped with security policy and measures according to the JIPDEC PrivacyMark ${ }^{\circledR}$ system, which is a widespread certification for companies managing personal information in Japan. The app's operating company, WIT Co. Ltd., has a secured data server in Japan to deal with all of the user information safely and appropriately while disclosing in the privacy policy what kinds of data are collected for the service and how and by whom the data can be used. In addition, the app data are stored and managed anonymously, kept re-identifiable only by the limited number of authorized researchers. Data managers store the file for such re-identification separately from the study data itself with password-protected access systems in an area with a limited access.

\section{Data Monitoring and Safety}

Clinical data of the participants will be extracted from electronic medical records and anonymously provided on an electronic CRF (eCRF) to the data manager. All entries in the CRFs should be backed up with the relevant source data, including informed consent forms, medical history, laboratory data, and recorded data on the app.

Monitoring of this research will be conducted by Innovative Clinical Research Center, Kanazawa University (iCREK). Monitors will ensure that the investigational team complies with the study protocol and that data and adverse events (AEs) are accurately recorded in the CRFs. Due to the low risk character of the intervention in this study, only spontaneously reported AEs will be reported, and the investigators will immediately take appropriate measures when AEs occur.

\section{Participant Timeline}

The schedule for enrollment, interventions, and assessments are shown in Fig. 4. Participants in either intervention group are seen at least once every 3 months for the collection of study outcome data. Data collection is performed during the patient's routine visits for diabetes checks.

\section{Sample Sizes}

The primary endpoint of this study is the change of HbA1c from baseline to endpoint (12 months of intervention). Based on the decrease in HbA1c in prior studies involving 


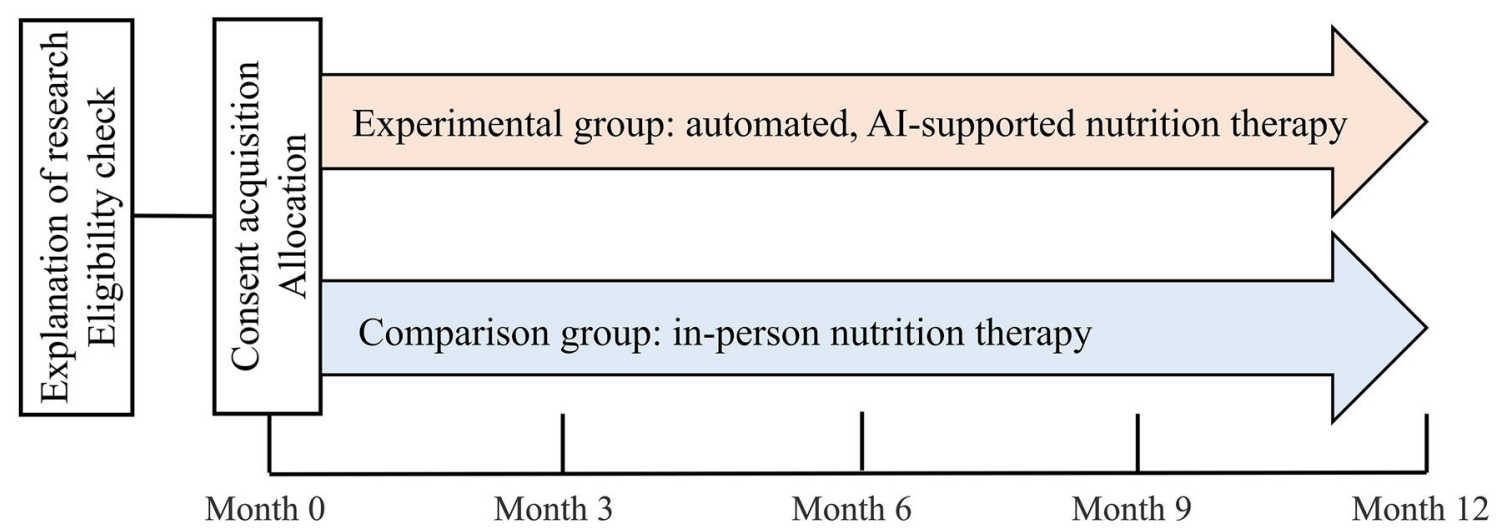

Fig. 4 Time schedule of the study. Patients who satisfy the eligibility criteria are randomized to two groups (1:1). In both groups, the principle of nutrition therapy is total energy restriction with macronutrient distributions

mobile-supported interventions [23], we expect that the mean change in HbA1c level will be $0.3 \%$ with a standard deviation of $0.3 \%$. At a significance level of 0.05 and a detection power of $80 \%$, the number of cases per group necessary to reach significant difference in change in HbA1c between two groups is calculated to be 74 cases. Assuming a $20 \%$ dropout rate, the target number of cases for each group is 50 cases, with a total of 100 participants in this study.

\section{Randomization and Blinding}

We use a web-based computerized blocked randomization with blocks of six to assign participants to either two modes of nutrition therapy. Block randomization is performed in each center separately. Randomization is conducted centrally and independently of investigators, and participants in each group are treated equally by their physicians. Although blinding of medical staff and participants to the study allocation is impossible, staff who are responsible for data collection are blinded.

\section{Statistical Analyses}

The primary objective of this study is to confirm that AI-supported, fully-automated nutritional intervention is not be inferior to in-person recommended by Japan Diabetes Society (JDS). Participants are seen once every 3 months for the collection of study outcome data. The observation period for both groups is 12 months

nutrition therapy by human dieticians with regard to HbA1c change during a 1-year period. Non-inferiority is considered confirmed with the use of a prespecified non-inferiority margin of $0.2 \%$. This is established if the lower limit of the $95 \%$ confidence interval of the difference in HbA1c change between the two groups (AIsupported group minus human-supported group) is $0.2 \%$. An independent $t$ test is used to compare the two groups for the change in HbA1c, which is calculated by subtracting the value of the baseline from the value after 1 year of intervention. The changes in secondary continuous outcomes of this study are also analyzed using an independent $t$ test. To account for non-dietary factors, such as the addition of new drugs, compliance to medications, and exercise habits, multiple regression analyses for changes in continuous outcomes are performed using these non-dietary factors as covariates.

To estimate the trajectories of repeated measurements for the study period, we use mixed-models. Differences between two groups (main effect) and the interaction between study groups and study month are assessed for outcomes, including changes in HbA1c, weight, BMI, lipid parameters, and blood pressure. All mixed models are adjusted for sex, age, baseline weight, and baseline HbA1c. Mixed models enable effective use of all available data even with missing values and, therefore, are 
considered an intention-to-treat method of analysis. Analyses are conducted using SPSS software version 24.0 for Windows (IBM Corp., Armonk, NY, USA). All tests are conducted as two-sided $\alpha=0.05$, and $95 \%$ confidence intervals are calculated.

\section{Participant Withdrawal}

Participants who discontinue using Asken or to seek advice according to the planned consultations with dieticians will continue to have data collected from their routine diabetes clinic visits, unless they specifically withdraw consent for this study.

\section{STRENGTHS AND LIMITATIONS}

This study is a randomized controlled trial with the aim to assess the efficacy of AI-supported automated nutrition intervention utilizing mobile technology on glycemic control in patients with type 2 diabetes mellitus. The study was designed to fill the information gap between the demand for mobile technologies promoting behavior change in eating habits and the scientific evidence supporting its efficacy compared to the conventional in-person interventions by humans [24].

The accuracy and validity of dietary evaluation is one of the strengths of this study. The dish-based nutritional evaluation performed by the Asken app has been validated to the weighed dietary records, which is the gold-standard method for individual level dietary assessment [25]. The correlation coefficients between those two methods have been reported to be 0.87 for total energy and $\geq 0.75$ for macronutrients, which are comparable or even higher than the correlation coefficients between the weighed dietary records and the food frequency questionnaire [15]. Because Asken has developed this database of $>100,000$ food items, which can even differentiate one kind of food at one shop from the similar kind at another place, those who often eat out or eat take-outs can more easily gain access to accurate nutritional evaluations.
Personalization of goal-settings and feedbacks are two additional strengths of AI-supported nutritional intervention and key components for the easy use of healthcare apps [26, 27]. Asken requests each patient to set his/her personalized goal for weight and behavior, and this function is expected to foster the intrinsic motivation, similar to other apps for self-management of chronic diseases $[28,29]$. The feedbacks are tailor-made based on the self-tracking data of each individual. Moreover, patients can customize food menus for their preferences, create their own items in the app, and cite a routine menu repeatedly on Asken. In their study analyzing the database from the app "Lose it," Serrano et al. reported that greater customization of the app is associated with a higher likelihood of engagement with the app and positive health outcomes [28]. Personalization of goals and feedbacks realized by use of AI and mobile technology is in line with the "personalized approach to diabetes management" that was recently highlighted in a joint position statement of the American Diabetes Association and the European Association for the Study of Diabetes [31].

There are some limitations to this study. First, because participants are limited to those who own and use a mobile phone, the results may not be generalized to generations with relatively lower information and communication technology literacy. Second, people who adhere to the healthcare apps are likely to have a deliberative style rather than intuitive style when making health-related decisions. Third, due to the unblinded design, the patients in the AI arm would know that they are getting the experimental intervention, which may result in better outcomes in the AI intervention than the control. Finally, Asken collects data not only on daily diets but also activities such as step counts. Independent contribution of nutritional intervention is to be evaluated by accounting for the effects of other wearable devices monitoring activities.

\section{ACKNOWLEDGEMENTS}

The authors thank the participants of the study, Chiyoko Takata and Yukiko Takamiya for their 
secretarial supports, and Ms. Eri Matsuzaki at Kagawa Nutrition University for her comments on methods.

Confidentiality. The study ID number is assigned at the time of registration and is used when handling samples and information related to the study to maintain anonymity. Using the study ID number as a key, electronic medical records, eCRFs, administrative forms, and recorded data on the app are linked. The lists that link study ID numbers to identifying information such as names or other personal identifiers are stored in a separate, locked file with password-protected access systems in an area with a limited access. All records that contain names or other personal identifiers, such as informed consent forms, are stored separately from the study records.

Funding. Sponsorship for this study and article processing charges are funded by Public Research of Kanazawa University Hospital. This study is also supported in part by Grantsin-Aid for Scientific Research from the Ministry of Education, Science, Sports, and Culture of Japan (17K09199).

Authorship. All named authors meet the International Committee of Medical Journal Editors (ICMJE) criteria for authorship for this article, take responsibility for the integrity of the work as a whole, and given their approval for this version to be published.

Author Contributions. MK and TY conceived the study. AN and KY provided advice on the study design and statistical analysis. YG participated in the discussion of study design and wrote the study plan. RO drafted the manuscript and AY critically revised the manuscript. All authors read and approved the final manuscript.

Compliance with Ethics Guidelines. The Institutional Review Board of the Kanazawa University approved the study protocol on April 13, 2018 (IRB no. 2623-3) in accordance with the ethical standards of the Declaration of Helsinki and current legal regulations in Japan. All procedures are in accordance with the ethical standards of the institutional and national committees responsible for human experimentation and with the Helsinki Declaration of 1964, as revised in 2013. Substantial amendments of the study protocol must be approved by the IRBs. The investigator provides each potential subject with an informed consent form that has been approved by the IRB, explains the contents of the study in detail both in writing and orally, and obtains the subject's written consent of his/her own free will.

Disclosures. Rie Oka has received a speaker fee from Novartis. Takashi Yoneda has received a research grant from Daiichi-Sankyo and speaker fees from Astellas, Novaritis, and Sanofi. Ayaka Yasugi is an employee of WIT Co., Ltd., Japan. Akihiro Nomura, Mitsuhiro Kometani, Yuko Gondoh, and Kenichi Yoshimura have nothing to declare.

Data Availability. Data sharing is not applicable to this article because it describes a study protocol.

Open Access. This article is distributed under the terms of the Creative Commons Attribution-NonCommercial 4.0 International License (http://creativecommons.org/licenses/ by-nc/4.0/), which permits any noncommercial use, distribution, and reproduction in any medium, provided you give appropriate credit to the original author(s) and the source, provide a link to the Creative Commons license, and indicate if changes were made.

\section{REFERENCES}

1. American Diabetes Association. 1. Strategies for improving care. Diabetes Care. 2016;39[Suppl 1]:S6-S12.

2. Knowler WC, Barrett-Connor E, Fowler SE, et al. Reduction in the incidence of type 2 diabetes with lifestyle intervention or metformin. N Engl J Med. 2002;346:393-403.

3. Pastors JG, Warshaw H, Daly A, Franz M, Kulkarni $\mathrm{K}$. The evidence for the effectiveness of medical 
nutrition therapy in diabetes management. Diabetes Care. 2002;25:608-13.

4. Eckel RH, Kahn SE, Ferrannini E, et al. Obesity and type 2 diabetes: what can be unified and what needs to be individualized? Diabetes Care. 2011;34:1424-30.

5. Beebe C. Body weight issues in preventing and treating type 2 diabetes. Diabetes Spectrum. 2003;16:261-6.

6. Pi-Sunyer X, Blackburn G, Brancati FL, et al. Reduction in weight and cardiovascular disease risk factors in individuals with type 2 diabetes: one-year results of the Look AHEAD trial. Diabetes Care. 2007;30:1374-83.

7. Esposito K, Maiorino MI, Petrizzo M, Bellastella G, Giugliano D. The effects of a mediterranean diet on the need for diabetes drugs and remission of newly diagnosed type 2 diabetes: follow-up of a randomized trial. Diabetes Care. 2014;37:1824-30.

8. Garden R. Expanding clinical empathy: an activist perspective. J Gen Intern Med. 2009;24:122-5.

9. Kluge EH. Resource allocation in healthcare: implications of models of medicine as a profession. MedGenMed. 2007;9:57.

10. Grock S, Ku JH, Kim J, Moin T. A review of technology-assisted interventions for diabetes prevention. Curr Diab Rep. 2017;17:107.

11. Bonoto BC, de Araujo VE, Godoi IP, et al. Efficacy of mobile apps to support the care of patients with diabetes mellitus: a systematic review and metaanalysis of randomized controlled trials. JMIR Mhealth Uhealth. 2017;5:e4.

12. Burke LE, Wang J, Sevick MA. Self-monitoring in weight loss: a systematic review of the literature. J Am Diet Assoc. 2011;111:92-102.

13. Burke LE, Conroy MB, Sereika SM, et al. The effect of electronic self-monitoring on weight loss and dietary intake: a randomized behavioral weight loss trial. Obesity (Silver Spring). 2011;19:338-44.

14. Boushey CJ, Spoden M, Zhu FM, Delp EJ, Kerr DA. New mobile methods for dietary assessment: review of image-assisted and image-based dietary assessment methods. Proc Nutr Soc. 2017;76:283-94.

15. Matsuzaki E, Michie M, Kawabata T. Validity of nutrient intakes derived from an internet website dish-based dietary record for self-management of weight among Japanese women. Nutrients. 2017;9:1058.
16. WHO Expert Consultation. Appropriate body-mass index for Asian populations and its implications for policy and intervention strategies. Lancet 2004;363:157-163.

17. Haneda $M$, Noda $M$, Origasa $H$, et al. Japanese clinical practice guideline for diabetes 2016. Diabetol Int. 2018;9:1-45.

18. Teramoto T, Sasaki J, Ueshima H, et al. Treatmenttherapeutic lifestyle modification. J Atheroscler Thromb. 2008;15:109-15.

19. Standard Tables of Food Composition in Japan. 2015. https://www.mext.go.jp/en/policy/science_ technology/policy/title01/detail01/1374030.htm. Accessed Feb 2019.

20. Ministry of Health, Labour and Welfare. Dietary reference intakes for Japanese. Daiichi Shuppan: Tokyo; 2015.

21. American Diabetes Association. 6. Glycemic targets: standards of medical care in diabetes-2019. Diabetes Care. 2019;42:S61-S70.

22. Oka R, Miura K, Sakurai M, et al. Comparison of waist circumference with body mass index for predicting abdominal adipose tissue. Diabetes Res Clin Pract. 2009;83:100-5.

23. Free C, Phillips G, Galli L, et al. The effectiveness of mobile-health technology-based health behaviour change or disease management interventions for health care consumers: a systematic review. PLoS Med. 2013;10:e1001362.

24. Wang Y, Xue H, Huang Y, Huang L, Zhang D. A systematic review of application and effectiveness of mHealth interventions for obesity and diabetes treatment and self-management. Adv Nutr. 2017;8:449-62.

25 Gibson R. Principles of nutritional assessment. Oxford University Press: Auckland; 2005

26. Lentferink AJ, Oldenhuis HK, de Groot M, Polstra L, Velthuijsen H, van Gemert-Pijnen JE. Key components in eHealth interventions combining selftracking and persuasive eCoaching to promote a healthier lifestyle: a scoping review. J Med Internet Res. 2017;19:e277.

27. Zhao J, Freeman B, Li M. Can mobile phone apps influence people's health behavior change? An evidence review. J Med Internet Res. 2016;18:e287.

28. Waterlander W, Whittaker R, McRobbie H, et al. Development of an evidence-based mHealth weight management program using a formative research process. JMIR Mhealth Uhealth. 2014;2:e18. 
29. Ramanathan N, Swendeman D, Comulada WS, Estrin D, Rotheram-Borus MJ. Identifying preferences for mobile health applications for self-monitoring and self-management: focus group findings from HIV-positive persons and young mothers. Int J Med Inform. 2013;82:e38-46.

30. Serrano KJ, Yu M, Coa KI, Collins LM, Atienza AA. Mining health app data to find more and less successful weight loss subgroups. J Med Internet Res. 2016;18:e154.

31. Inzucchi SE, Bergenstal RM, Buse JB, et al. Management of hyperglycemia in type 2 diabetes: a patient-centered approach. Position statement of the American Diabetes Association (ADA) and the European Association for the Study of Diabetes (EASD). Diabetes Care 2012; 35:1364-79. 